\title{
Prevalence, Antibiotic Susceptibility and Diversity of Vibrio parahaemolyticus Isolates in Seafood from South China
}

\author{
Ying Yang, Jiafang Xie, Hua Li, Shuwen Tan, Yanfeng Chen and Hui Yu* \\ School of Life Science and Engineering, Foshan University, Foshan, China
}

Vibrio parahaemolyticus is a leading cause of foodborne infections in China and a threat to human health worldwide. The main objective of this study is to determine the prevalence and characteristic of $V$. parahaemolyticus isolates in fish, oyster and shrimp samples from the South China domestic consumer market. To accomplish this, we examined 504 seafood samples from 11 provinces of China. The prevalence rates were 9.38, 30.36, and $25.60 \%$, respectively. In summer (33.33\%), the prevalence of V. parahaemolyticus was more common than that detected in the winter (14.01\%).

OPEN ACCESS

Edited by: Aldo Corsetti, Università di Teramo, Italy

Reviewed by: Anwar Huq University of Maryland, College Park, United States

Ben D. Tall,

U.S. Food and Drug Administration,

United States

*Correspondence:

Hui Yu

yu88hui@qq.com

Specialty section:

This article was submitted to

Food Microbiology,

a section of the journal

Frontiers in Microbiology

Received: 27 September 2017 Accepted: 11 December 2017

Published: 20 December 2017

Citation:

Yang Y, Xie J, Li H, Tan S, Chen Y and Yu H (2017) Prevalence, Antibiotic Susceptibility and Diversity of Vibrio parahaemolyticus Isolates

in Seafood from South China.

Front. Microbiol. 8:2566.

doi: 10.3389/fmicb.2017.02566
In addition, we identified $98 \mathrm{~V}$. parahaemolyticus strains. The antimicrobial resistance trends of our seafood isolates to 15 antimicrobial agents revealed that major isolates were resistant to ampicillin (79.59\%). Furthermore, $68.38 \%$ of the isolates were identified as being multidrug resistance. The prevalence of $t d h$ or trh genes among the isolates was 8.16 and 12.24\%, respectively. ERIC-PCR and multilocus sequence typing (MLST) results enabled classification of the isolates $(n=98)$ into different clusters, revealing genetic variation and relatedness among the isolates. Thus, our findings demonstrate the prevalence of $V$. parahaemolyticus in a variety of common seafood consumed domestically in China and provides insights into the dissemination of antibioticresistant strains, which should improve our microbiological risk assessment knowledge associated with $V$. parahaemolyticus in seafoods.

Keywords: Vibrio parahaemolyticus, prevalence, antibiotic resistance, virulence gene, MLST

\section{INTRODUCTION}

Foodborne disease remains a threat to public health worldwide. Vibrio parahaemolyticus is a halophilic Gram-negative bacterium that is commonly associated with food-borne gastroenteritis. This bacterium has been found in seawater, marine fish, shellfish, etc. (Fujino et al., 1953; Joseph et al., 1982; Kang et al., 2016) and causes outbreaks worldwide. In China, V. parahaemolyticus is also an important cause of food poisoning associated with consumption of seafood ( $\mathrm{Yu}$ et al., 2015; Tan et al., 2017). It was also reported that the population levels of V. parahaemolyticus tends to show strong seasonal trends (Shen et al., 2010). Consequently, foodborne outbreaks caused by $V$. parahaemolyticus typically show a seasonal difference, peaking in the warmer months of the United States (Daniels et al., 2000). However, to date, the presence and contamination levels of $V$. parahaemolyticus in seafood from different seasons has received less attention, and little information is available among various types of samples. 
As a result of the excessive use of antibiotics in human and aquaculture systems, some antibiotics have no longer been effective in controlling pathogens infections during the past several decades (Elmahdi et al., 2016). Variation in antibiotic resistance patterns among $V$. parahaemolyticus strains isolated from different countries and sources has been observed (Odeyemi and Stratev, 2016). Recent studies regarding a $V$. parahaemolyticus isolate demonstrated resistance to certain common antibiotics such as ampicillin and kanamycin (Lopatek et al., 2015; Xie et al., 2015). The frequent occurrence of multi-drug resistance in $V$. parahaemolyticus is among the most important of public health concerns (Xie et al., 2017). In order to improve yield and treatment of infectious diseases in farm products, increase levels of antibiotics in animal feed and water is a common behavior (Letchumanan et al., 2015a). Therefore, it is very important to set up a monitoring system to research on antimicrobial-resistance trends.

An important virulence genes, toxR is involved in the regulation of many genes in $V$. parahaemolyticus (Lin et al., 1993). The pathogenicity of $V$. parahaemolyticus is strongly correlated with the production of either thermostable direct hemolysin $(\mathrm{TDH}), \mathrm{TDH}$ related hemolysin $(\mathrm{TRH})$, or both (Honda and Iida, 1993; Mala et al., 2016). V. parahaemolyticus strains isolated from diarrheal patients produce thermostable $\mathrm{TDH}$ which is a toxin with several biological properties, including hemolytic activity, enterotoxicity and cytotoxicity, $\mathrm{TDH}$ is also present in most of the Kanagawa phenomenon (KP)-positive clinical strains. TRH is believed to act similarly to $\mathrm{TDH}$. (Takahashi et al., 2000; Matsuda et al., 2010; Leoni et al., 2016). Presently, PCR-based methods is useful in identified of $t d h$ and trh gene in $V$. parahaemolyticus isolates (Shirai et al., 1990).

The utility of molecular techniques has already been established in epidemiological studies of $V$. parahaemolyticus infections, such as pulsed-field gel electrophoresis (PFGE) (Marshall et al., 1999) and random amplified polymorphic DNA (RAPD) analysis (Yang et al., 2008). Enterobacterial repetitive intergenic consensus sequence PCR (ERIC-PCR) is an easy, fast, and relatively cheap method that is comparable to PFGE in terms of its discriminatory power and reproducibility, It is also superior to other PCR-based fingerprinting techniques (Wong and Lin, 2001; Jun et al., 2012). Multilocus sequence typing (MLST) is based on sequence analysis of housekeeping $(\mathrm{HK})$ genes and is a useful method to trace the global epidemiology of bacterial pathogens. MLST, which is sequence based, provides a clear determination of the genetic features of strains that is consistent from one laboratory to another. Previous MLST studies of $V$. parahaemolyticus isolates have provided a better understanding of the genetic relatedness within these bacteria. These studies also revealed the relative evolutionary importance of mutations and lateral gene transfer events among these strains (Gonzálezescalona et al., 2008; Banerjee et al., 2014).

Overall, $V$. parahaemolyticus strains are often isolated from fish, shrimp and other types of seafoods. Recently, $V$. parahaemolyticus was also found in ready to eat food (Cho et al., 2016; Mala et al., 2016). In the Zhejiang province of China alone, there were 71 outbreaks caused by $V$. parahaemolyticus resulting in 933 illnesses and 117 hospitalizations from 2010 to 2014. In our study, we analyzed 224 fish, 112 oyster, and 168 shrimp samples. In China, seafood is very popular and higher consumption is correlated with an increase in the overall standard of living in the population. Our study aimed to find the differences in the seasonal prevalence of $V$. parahaemolyticus in all provinces of South China. We next characterized the prevalence all of the identified isolates to determine their genetic relatedness by phenotyping and genotyping methods. We attempted to confirm the virulence and antibiotic resistance trends. We also performed ERIC and MLST typing of the isolated strains, which revealed the molecular diversity of the $V$. parahaemolyticus isolates. These findings serve as baseline values that support the establishment of a national seafood surveillance system to ensure food safety in China.

\section{MATERIALS AND METHODS}

\section{Sample Collection}

From July 2015 to July 2017, a total of 504 aquatic products samples, including 224 fish samples, 112 oyster samples and 168 shrimp samples were collected from retail markets from 14 different cities in China, belonging to 11 provinces (Figure 1). In these regions, the climate is cold from September to March (winter), and it is hot from March to September (summer). From each city, we collected eight fish samples, four oyster samples, and six shrimp samples (from four different retail markets) during both summer and winter months. We put the samples in sterile sealed plastic bags and sent them to the lab in an ice box $\left(4^{\circ} \mathrm{C}\right)$. Samples were analyzed immediately thereafter.

\section{Detection and Enumeration of V. parahaemolyticus}

The prevalence and bacterial load of $V$. parahaemolyticus in the samples were tested using the most probable number (MPN) method according to the National Food Safety Standards of China (GB4789.7-2013); this method was reported previously by Xie et al. (2016). In brief, we first weighed and homogenized $25 \mathrm{~g}$ of each sample. Then, $225 \mathrm{~mL}$ of alkaline peptone water (APW) with $3 \% \mathrm{NaCl}$ was added; $3 \times 1 \mathrm{~mL}$ of each sample dilution was inoculated into $9 \mathrm{~mL}$ of APW (3\% $\mathrm{NaCl}$ ) when prepared up to a 1:1000 dilution. Samples were incubated at $37^{\circ} \mathrm{C}$ for $18 \mathrm{~h}$. After that, we used an inoculation loop to streaked the solution onto thiosulfate-citrate-bile saltssucrose (TCBS) agar plates and incubated them at $37^{\circ} \mathrm{C}$ for $24 \mathrm{~h}$. The $\mathrm{r}$ green or blue green colonies of $2-3 \mathrm{~mm}$ in diameter were presumed to be $V$. parahaemolyticus isolates, three to five colonies (if present) were chose from each plate, streaked onto Chromogenic Vibrio Medium and culture at $37^{\circ} \mathrm{C}$ for $24 \mathrm{~h}$. One (if present) colony (mauve) from each Chromogenic Vibrio Medium plate was selected for further identification tests, such as Gram staining, $\mathrm{NaCl}$ tolerance tests, oxidase activity and analysis with API 20E diagnostic strips (BioMerieux, Marcy-l'Étoile, France). The pollution result in the 


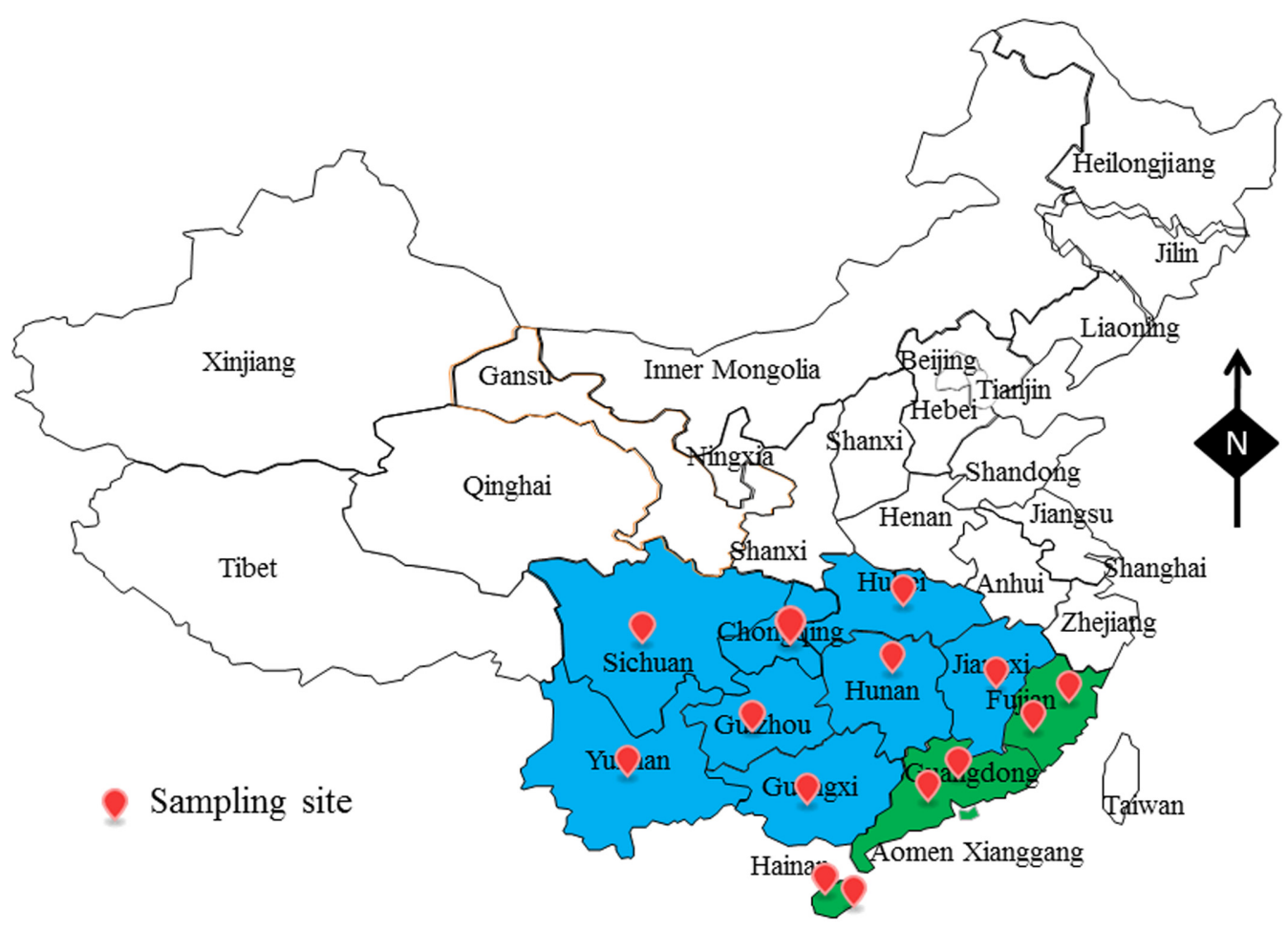

FIGURE 1 | The sampling site of foods sample in South China.

samples were determined using an MPN table. The levels of contamination in the samples were determined using an MPN table.

\section{Antimicrobial Susceptibility Testing}

The susceptibility of the $V$. parahaemolyticus isolates to antibiotics was examined by the disk-diffusion method, according to the guidelines of the Clinical and Laboratory Standards Institute (CLSI, 2012; Miller et al., 2014). Muller Hinton agar and a panel of 15 antibiotics disks were selected for the resistance analysis. The 15 common antimicrobials used in this study belonged to six classes and were as follows: $\beta$-lactam [ampicillin: AMP (10 $\mu \mathrm{g})$; piperacillin: PRL $(20 \mu \mathrm{g})$; cefotaxime: CTX $(30 \mu \mathrm{g})$; cefoxitin: FOX $(30 \mu \mathrm{g})$; cephazolin: KZ (30 $\mu \mathrm{g})$; imipenem: IPM (20 $\mu \mathrm{g})$; meropenem: MEM (20 $\mu \mathrm{g})]$, aminoglycoside [gentamicin: CN (10 $\mu \mathrm{g})$; kanamycin: $\mathrm{K}$ $(30 \mu \mathrm{g})$; streptomycin: $\mathrm{S}(10 \mu \mathrm{g})$ ], tetracycline [tetracycline: TET (30 $\mu \mathrm{g})$ ], quinolone [ciprofloxacin:CIP (5 $\mu \mathrm{g})$; levofloxacin: LEV $(20 \mu \mathrm{g})$ ], sulfonamides [trimethoprim-sulfamethoxazole: SXT $(25 \mu \mathrm{g})$ ], chloramphenicol [chloramphenicol:C $(30 \mu \mathrm{g})$ ]. Following the methods of the CLSI, sensitive (S), intermediate (I), or resistant (R) classification system was used.

\section{Detection of Virulence Genes: toxR, $t d h$, and $t r h$}

According to the manufacturer's instructions, genomic DNA was extracted from each $V$. parahaemolyticus isolate using a bacterial DNA extraction kit (Sangon, Shanghai, China).
The toxR gene is important and appears to be well conserved among $V$. parahaemolyticus isolates. The primers were as follows F: GTCTTCTGACGCAATCGTTG, R: ATACGAGTGGTTGCT GTCATG (Kim et al., 1999). The $t d h$ and $t r h$ genes detection was executed as previously reported (West et al., 2013). The following primers were used: Tdh-F:CTGTCCCTTTTCCTGCCCCCG, Tdh-R:AGCCAGACACCGCTGCCATTG;Trh-F:ACCTTTTCC TTCTCCWGGKTCSG,Trh-F:CCGCTCTCATATGCYTCGACA $\mathrm{KT}$ ). All the oligonucleotide primers were synthesized by Sangon Biotech (Shanghai, China). The PCR reaction system contained DNA template $(1 \mu \mathrm{L}), 0.5 \mu \mathrm{M}$ of each primer, $12.5 \mu \mathrm{L}$ of $: 2 \times$ PCR mix (Qiagen), and $\mathrm{ddH}_{2} \mathrm{O}(9.5 \mu \mathrm{L})$. The amplified thermal-cycling program was set with the following conditions: denaturation at $95^{\circ} \mathrm{C}(5 \mathrm{~min}) ; 40$ cycles of $95^{\circ} \mathrm{C}$ for $1 \mathrm{~min}$, $62^{\circ} \mathrm{C}$ for $1 \mathrm{~min}$, and $72^{\circ} \mathrm{C}$ for $1 \mathrm{~min}$; and a final extension step of $72^{\circ} \mathrm{C}$ for $5 \mathrm{~min}$. PCR amplicons were electrophoresed on $2.0 \%$ (wt/vol) agarose gels containing GoldView. The images were captured digitally and analyzed using a gel imaging system. $V$. parahaemolyticus strains ATCC33847 $(t d h+)$ and ATCC17802 $(t r h+)$ were used as positive controls, and distilled water was used as a negative control.

\section{ERIC-PCR Analysis}

ERIC-PCR analysis was performed on the $V$. parahaemolyticus isolates using a previously reported method (Chen et al., 2012; Xie et al., 2015). A pair of primers, ERIC - F (5-ATGTAAGCTCCTGGGGATTCAC-3) and ERIC -R (5AAGTAAGTGACTGGGGTGAGCG-3) were used. ERIC-PCR 
TABLE 1 | Prevalence of Vibrio parahaemolyticus in food samples from South China.

\begin{tabular}{|c|c|c|c|c|c|}
\hline \multirow[t]{2}{*}{ Samples } & \multirow{2}{*}{$\begin{array}{c}\text { Number of } \\
\text { samples analyzed }\end{array}$} & \multirow{2}{*}{$\begin{array}{l}\text { Number of samples } \\
\text { positive (\%) }\end{array}$} & \multicolumn{3}{|c|}{ Number of samples containing the pathogen } \\
\hline & & & $<3(\mathrm{MPN} / \mathrm{g})$ & $3-100$ (MPN/g) & $>100-10^{3}(\mathrm{MPN} / \mathrm{g})$ \\
\hline Fish & 224 & 21 (9.38) & 3 & 13 & 5 \\
\hline Oyster & 112 & $34(30.36)$ & 8 & 16 & 10 \\
\hline Shrimp & 168 & 43 (25.60) & 6 & 33 & 4 \\
\hline Total & 504 & 98 (19.44) & 17 & 62 & 19 \\
\hline
\end{tabular}

TABLE 2 | Prevalence of Vibrio parahaemolyticus during different seasons.

\begin{tabular}{|c|c|c|c|c|c|}
\hline \multirow[t]{2}{*}{ Samples } & \multirow{2}{*}{$\begin{array}{c}\text { Number of } \\
\text { samples analyzed }\end{array}$} & \multirow{2}{*}{$\begin{array}{c}\text { Number of samples } \\
\text { positive (\%) }\end{array}$} & \multicolumn{3}{|c|}{ Number of samples containing the pathogen } \\
\hline & & & $<3(\mathrm{MPN} / \mathrm{g})$ & $3-100$ (MPN/g) & $>100-10^{3}(\mathrm{MPN} / \mathrm{g})$ \\
\hline Summer & 207 & 69 (33.33) & 9 & 46 & 14 \\
\hline Winter & 207 & $29(14.01)$ & 8 & 16 & 5 \\
\hline Total & 905 & 98 (19.44) & 17 & 62 & 19 \\
\hline
\end{tabular}

typing was performed as follows: $1 \mu \mathrm{l}$ of genomic DNA (100 ng) was added to a master mixture that contained $0.6 \mu \mathrm{mol} / \mathrm{L}$ of each primer, $12.5 \mu \mathrm{L}$ of $2 \times$ Long Taq mix (Dongsheng Biotech, Guangzhou, China), and $\mathrm{ddH}_{2} \mathrm{O}$ to a total volume of $25 \mu \mathrm{L}$ per reaction. After an initial denaturation at $95^{\circ} \mathrm{C}$ for $5 \mathrm{~min}, 35$ cycles of amplification were performed under the following conditions: denaturation at $94^{\circ} \mathrm{C}$ for $45 \mathrm{~s}$, annealing $52^{\circ} \mathrm{C}$ for $1 \mathrm{~min}$ and extension at $72^{\circ} \mathrm{C}$ for $3 \mathrm{~min}$ followed by a final extension step at $72^{\circ} \mathrm{C}$ for $10 \mathrm{~min}$. The amplicons were electrophoresed on $2.0 \%$ (wt/vol) agarose gels containing GoldView. The images were captured digitally in TIFF file format for further analysis.

\section{MLST Analysis}

The $V$. parahaemolyticus MLST website and database ${ }^{1}$ shows the MLST analysis procedure (Jolley et al., 2004). Seven genes were selected (recA, gyrB, dnaE, dtdS, pntA, pyrC, and tnaA). The amplified thermal-cycling program was designed with the following conditions: denaturation at $95^{\circ} \mathrm{C}(5 \mathrm{~min}) ; 30$ cycles of $95^{\circ} \mathrm{C}$ for $1 \mathrm{~min}, 58^{\circ} \mathrm{C}$ for $1 \mathrm{~min}$, and $72^{\circ} \mathrm{C}$ for $1 \mathrm{~min}$; and a final extension step of $72^{\circ} \mathrm{C}$ for $10 \mathrm{~min}$. A BGI instrument (Shenzhen, China) was used to sequence the PCR amplicons. The assigning allele numbers and defined sequence types (STs) ${ }^{1}$. BioEdit was used to determine the alignments of these sequences.

\section{Statistical Analysis}

The size of each band in the ERIC patterns was determined and the data were coded as 0 (absent) or 1 (present). Cluster analysis was performed with NTSYS-pc (version 2.10), a numerical taxonomy and multivariate analysis software package (Rohlf, 2000), based on Dice's similarity coefficient (SD), with a $1 \%$ position tolerance and the unweighted-pair group method with arithmetic averages (UPGMA). The evolution tree of the concatenated sequences of the seven loci (MLST) was built based on the method involving the Kimura-2-parameter in Mega 6.0 (Tamura et al., 2013).

\footnotetext{
${ }^{1}$ http://pubmlst.org/vparahaemolyticus/
}

\section{RESULTS}

\section{Prevalence and Level of V. parahaemolyticus}

Of the 504 seafood samples that we tested, 98 (19.44\%) were identified as $V$. parahaemolyticus positive. This included 21 (9.38\%) of the 224 fish samples, 34 (30.36\%) of the 112 oyster samples and $43(25.60 \%)$ of the 168 shrimp samples. These results are shown in Table 1. Overall, the degree of $V$. parahaemolyticus contamination in these samples varied from 1.50 to $1000 \mathrm{MPN} / \mathrm{g}$. Most of the positive samples had a level of 3 to $10 \mathrm{MPN} / \mathrm{g}(62 / 98,63.27 \%)$. Moreover, $19.39 \%$ (19/98) of the positive samples exceeded $100 \mathrm{MPN} / g$, and 17 samples were below $3 \mathrm{MPN} / \mathrm{g}$. In total, $98 \mathrm{~V}$. parahaemolyticus isolates were confirmed (Supplementary Table S1).

Considering the influence of the season, the number of positive summer samples was much higher than the number of positive winter samples (Table 2). The isolation rate of $V$. parahaemolyticus in summer reached $33.33 \%$, whereas it was $14.01 \%$ in the winter. Moreover, the $V$. parahaemolyticus population differed significantly for samples collected during the winter and summer. Densities among samples collected in the summer varied less, and the contamination level was higher than that observed in the winter samples. In summer, the mean levels of $V$. parahaemolyticus in samples were $99.08 \mathrm{MPN} / \mathrm{g}$, which is higher than that observed in the winter $(22.13 \mathrm{MPN} / \mathrm{g})$. A total of 14 samples with densities above $100 \mathrm{MPN} / \mathrm{g}$ were detected.

\section{Antimicrobial Susceptibility of the V. parahaemolyticus Isolates}

Isolates of $V$. parahaemolyticus were tested for antibiotic susceptibility, the resistance patterns to 15 antibiotics are shown in Table 3. The isolates were most resistant to ampicillin, with resistance and intermediate rates of 79.59 and $12.24 \%$, respectively. In addition, the isolates exhibited relatively high resistance rates, of $68.37,39.80$, and $39.80 \%$, for streptomycin, 
TABLE 3 | Antimicrobial resistance profiles of Vibrio parahaemolyticus isolates.

\begin{tabular}{lccc}
\hline $\begin{array}{l}\text { Antimicrobial } \\
\text { agents }\end{array}$ & \multicolumn{3}{c}{ Vibrio parahaemolyticus $(\boldsymbol{n}=\mathbf{9 8})$} \\
\cline { 2 - 4 } & $\begin{array}{c}\text { Number } \\
\text { (\%) of R }\end{array}$ & $\begin{array}{c}\text { Number } \\
\text { (\%) of I }\end{array}$ & $\begin{array}{c}\text { Number } \\
\text { (\%) of S }\end{array}$ \\
\hline Ampicillin (AMP) & $78(79.59)$ & $12(12.24)$ & $8(8.16)$ \\
Piperacillin (PRL) & $8(8.16)$ & $1(1.02)$ & $89(90.82)$ \\
Cefotaxime (CTX) & $0(0.00)$ & $2(2.04)$ & $96(97.96)$ \\
Cefoxitin (FOX) & $0(0.00)$ & $1(1.02)$ & $97(98.98)$ \\
Cefazolin (KZ) & $39(39.80)$ & $18(18.37)$ & $41(41.84)$ \\
Imipenem (IPM) & $0(0.00)$ & $2(2.04)$ & $96(97.96)$ \\
Meropenem (MEM) & $0(0.00)$ & $0(0.00)$ & $98(100.00)$ \\
Gentamicin (CN) & $17(17.35)$ & $20(20.41)$ & $61(62.24)$ \\
Kanamycin (K) & $39(39.80)$ & $24(24.49)$ & $35(35.71)$ \\
Streptomycin (S) & $67(68.37)$ & $26(26.53)$ & $5(5.10)$ \\
Tetracycline (TE) & $13(13.27)$ & $8(8.16)$ & $77(78.57)$ \\
Ciprofloxacin (CIP) & $5(5.10)$ & $2(2.04)$ & $91(92.86)$ \\
Levofloxacin (LEV) & $1(1.02)$ & $5(5.10)$ & $92(93.88)$ \\
Trimethoprim- & $9(9.18)$ & $12(12.24)$ & $77(78.57)$ \\
sulfamethoxazole & & & \\
(SXT) & & & $95(96.94)$ \\
Chloramphenicol (C) & $2(2.04)$ & $1(1.02)$ & \\
\hline
\end{tabular}

cefazolin, and kanamycin, respectively. Fortunately, all the examined isolates were susceptible to cefotaxime, cefoxitin, imipenem and meropenem. Among the remaining tested antibiotics, the isolates were partially susceptible to piperacillin, ciprofloxacin, levofloxacin, or chloramphenicol. However, among all the isolates, there were multidrug-resistant isolates (Vps15) showing resistance to seven antibiotics and some strains showing resistance to five antibiotics. In addition, 68.38\% (67/98) of the isolates were resistance to more than three antibiotics (Supplementary Table S1).

\section{The toxR, tdh, and trh Genes Test}

To detect pathogenic isolates, all of the $98 \mathrm{~V}$. parahaemolyticus isolates were examined for the presence of the toxR, trh, and $t d h$ genes. This analysis is summarized in Table 4. All of the isolates were positive for tox R. Among them, 8.16\% (8/98) and $12.24 \%$ (12/98) of the $V$. parahaemolyticus strains carried the $t d h$, or trh gene, respectively; in contrast, none of the isolates harbored both the $t d h$ and trh genes. For the $t d h$-positive strains, two were found in a fish source, two were identified from oysters, and three were found from shrimp. Of the trh-positive isolates, five samples were from fish, four samples were from oyster, and three samples were from shrimp.

\section{ERIC-PCR and MLST}

Figure 2 shows the results from ERIC-PCR analysis of the 98 isolates. There were 5 to 10 amplification bands, with sizes ranging between $100 \mathrm{bp}$ to about $6000 \mathrm{bp}$. Analysis of the ERIC-PCR patterns revealed that those strains could be divided into six clusters, A, B, C, D, E, and F at a relative similarity coefficient of 0.67 . The isolates proved to be very diverse genetically. Most isolates were distributed among the A and D clusters. However,
TABLE 4 | Distribution of the tox $R$, tdh, and trh genes in $V$. parahaemolyticus isolates.

\begin{tabular}{lcccc}
\hline $\begin{array}{l}\text { Sample } \\
\text { category }\end{array}$ & $\begin{array}{c}\text { Number of } \\
\text { isolates }\end{array}$ & $\begin{array}{c}\text { tox } \\
\boldsymbol{R} \text {-positive } \\
(\%)\end{array}$ & $\begin{array}{c}\boldsymbol{t} \boldsymbol{\text { th }} \text { - positive } \\
\mathbf{( \% )}\end{array}$ & $\begin{array}{c}\boldsymbol{t} \boldsymbol{\text { trh-positive }} \\
\mathbf{( \% )}\end{array}$ \\
\hline Fish & 21 & $100(21 / 21)$ & 2 & 5 \\
Oyster & 34 & $100(34 / 34)$ & 2 & 4 \\
Shrimp & 43 & $100(43 / 43)$ & 4 & 3 \\
Total & 98 & $100(98 / 98)$ & $8.16(8 / 98)$ & 12.24 \\
& & & & $(12 / 98)$ \\
\hline
\end{tabular}

we did not find a relationship between the different sample types and the ERIC-PCR clusters

The isolates were analyzed by MLST using the sequences generated from the internal fragments of the seven HK genes. The alleles numbers and STs were assigned according to a database created for $V$. parahaemolyticus isolates upon submitting the sequence results. A total of 86 STs were observed among the 98 isolates. The numbers of alleles observed for each MLST locus in this study showed statistics as follows: dna E: 61; gyr B: 70; rec A: 62; dtd S: 57; pnt A: 43; pyr C: 60; and tna A: 42. The haplotype diversity was 0.987 . A minimum evolutionary tree was constructed using the concatenated sequences of each allele. The MLST results divided the V. parahaemolyticus isolates into five clusters (designated as I, II, III, IV, and V). Most isolates were distributed in cluster I. Interestingly, Figure 3 shows that all the isolates in cluster III were from Fujian, whereas, cluster IV contained the $V$. parahaemolyticus isolates from Hainan. In cluster $\mathrm{V}$, most of the isolates were from three coastal cities. For strain Vps18 and Vps06 (from Guangdong), the ST type (ST212) showed a greater distance on the evolutionary tree.

\section{DISCUSSION}

According to epidemiologic reports, V. parahaemolyticus is a major cause of bacterial infections associated with the consumption of raw or undercooked aquatic products including different kinds of shellfish and fish (Tan et al., 2017). Our results show that the prevalence of $V$. parahaemolyticus was higher in oyster samples $(30.36 \%)$ than in the other seafood samples. This prevalence is also lower than that found in Germany (41.8\%) (Jones et al., 2012). The prevalence of $V$. parahaemolyticus isolates detected off the eastern coast of China was also reported to be higher than that observed in our study (Zhao et al., 2011). The reason for this discrepancy maybe that most of the sampled cities were inland, with much fewer kinds of seafood. Consistent with this hypothesis, the prevalence of this bacteria in coastal cities (Guangdong, Hainan, and Fujian) was higher (Supplementary Table S1).

Notably, the prevalence of $V$. parahaemolyticus in summer (33.33\%) was higher than that in winter (14.01\%). Our results are in accordance with previous reports that $V$. parahaemolyticus outbreaks typically show a seasonal pattern, peaking in the warmer months (Daniels et al., 2000; Chen et al., 2017), and that $V$. parahaemolyticus isolates are cold sensitive, thus, lower 

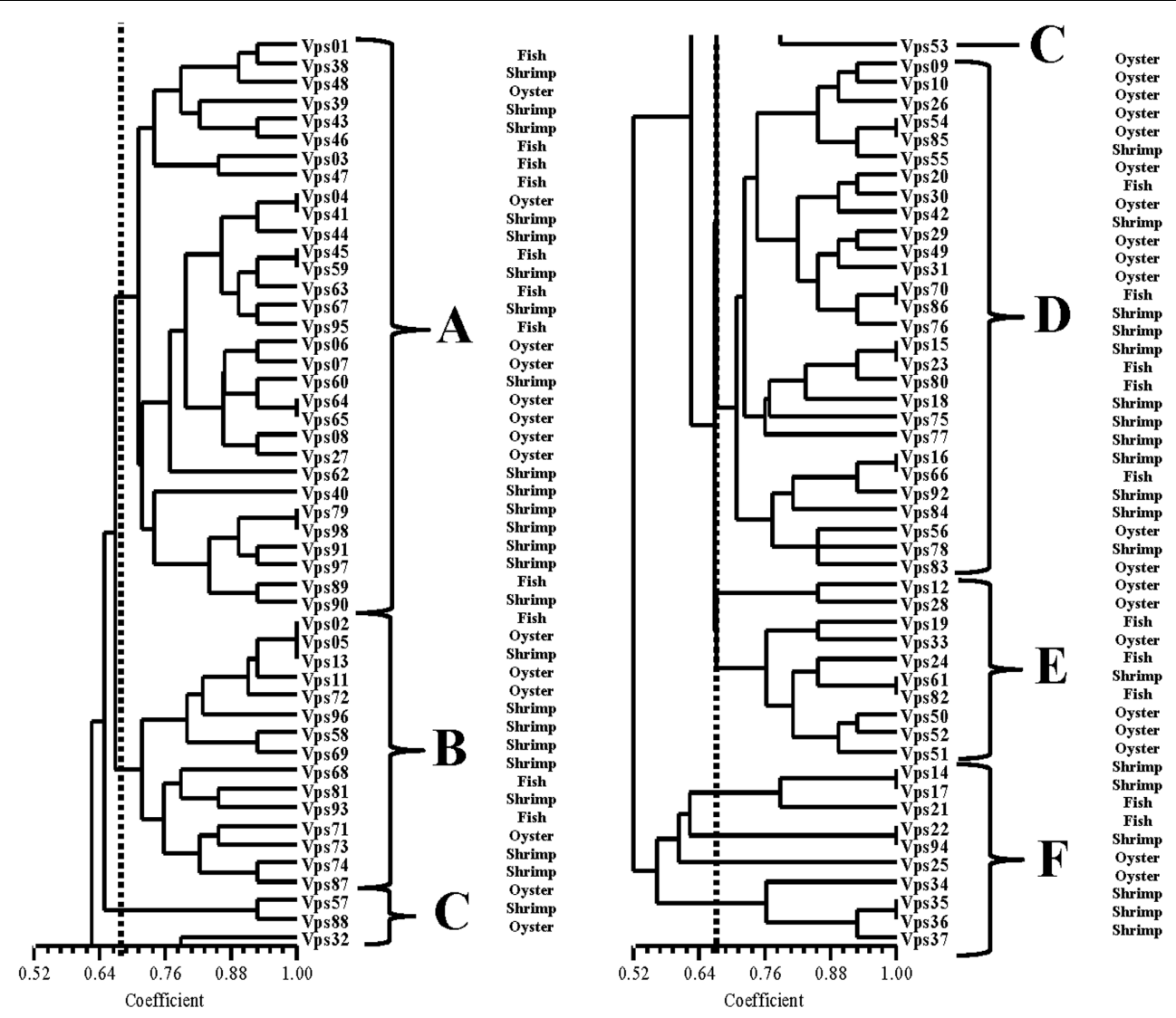

FIGURE 2 | ERIC-PCR DNA fingerprint analysis of $V$. parahaemolyticus isolates.

temperature in winter may limit or reduce their growth ( $\mathrm{Su}$ and Liu, 2007). As our results were not only obtained from samples collected from a majority of regions in South China, but also from different seasons and kinds of seafood, the data can be useful for determining optimal temperature values for control of $V$. parahaemolyticus contamination (World Health Organization, 2014).

As there is an increase in the number of resistance genes and the spread of antimicrobial-resistant $V$. parahaemolyticus isolates worldwide, the misuse and overuse of antibiotics are considered the most important factors (Yano et al., 2014; Tan et al., 2016). In the Hunan province of China, the average utilization rate of antibiotics reached $64.56 \%$ and more than 50 antibiotics were available for sale (Daimei and Dai, 2016). Susceptibility tests show that isolates $V$. parahaemolyticus in South China appear to a high level of resistance to ampicillin. This result is similar to a report by Letchumanan et al. (2015b) in which $82 \%$ of the isolates from shrimp samples were also resistant to ampicillin. In addition, many isolates were resistant to streptomycin (68.37\%) and kanamycin (39.80\%). Similarly, previous studies have suggested that resistance to these antibiotics is common in $V$. parahaemolyticus isolates (Elexson et al., 2014; Shaw et al., 2014). Susceptibility profiles to antibiotic classes such as cefotaxime, cefoxitin, imipenem, and meropenem were examined. Notably, we found some isolates showing resistance to chloramphenicol, tetracycline or ciprofloxacin, which are first-line drugs used in clinical treatment (Ottaviani et al., 2013; Elmahdi et al., 2016). In this work, 68.38\% of the strains were multi-drug resistant and some even showed resistance to seven antibiotics. This ratio is higher than that detected in previous reports (Letchumanan et al., 2015a; Xu et al., 2016). In general, evaluating variations in the antimicrobial susceptibility trends of $V$. parahaemolyticus strains is important give that infection emergence of microbial resistance to multiple drugs is a serious clinical problem.

The toxR gene, which is involved in the regulation of many other genes, was ubiquitous in $V$. parahaemolyticus isolates. As we know, not all $V$. parahaemolyticus strains are pathogenic in humans; however, the products of the hemolysin genes ( $t d h$ and trh) are believed to rapidly induce inflammatory gastroenteritis and are often detected in clinical strains (Mahoney et al., 2010; Raghunath, 2015). Thus, detection of the hemolysin genes could be an important way to infer the virulence potential of food isolates. In our study, up to 8.16 and $12.24 \%$ of the strains were $t d h$ or trh positive. Our findings are similar to those of previous studies on environmental $V$. parahaemolyticus isolates (Gutierrez West et al., 2013; Letchumanan et al., 2015a). The distributions and evolution of $t d h+$ and/or trh + strains 

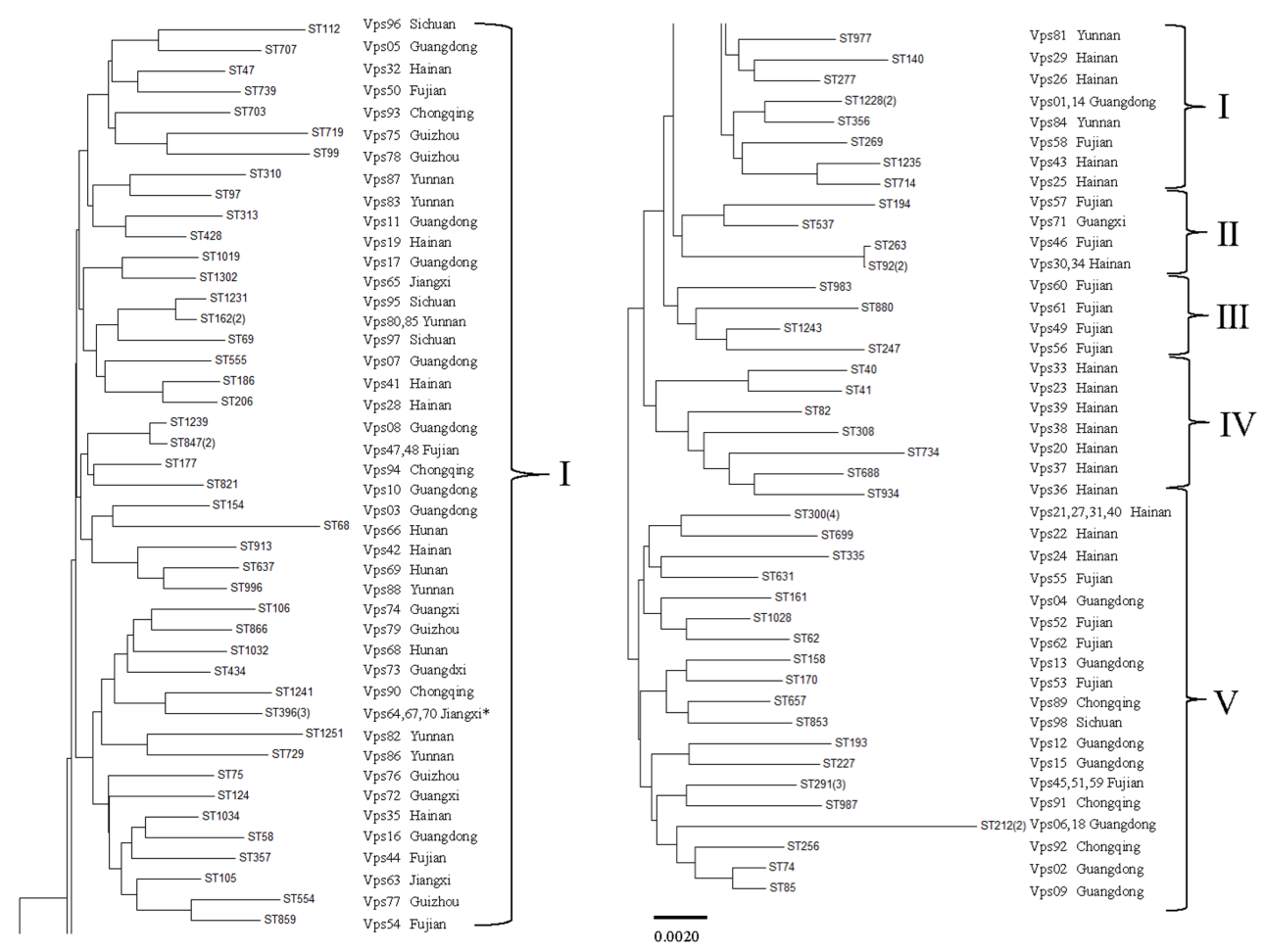

FIGURE 3 | Multilocus sequence typing (MLST) minimum evolution tree of V. parahaemolyticus isolates (“*” stand for "Jiangxi, Hunan, Guangxi”).

may differ depending on sample source, geographical region, and other environmental factors (Wilson and Salyers, 2003; Raghunath, 2015). However, the relatively high percentages of $t d h$ and trh positive $V$. parahaemolyticus isolates identified in food samples represent a potential public health risk.

Recently, the utility of molecular techniques has been established in epidemiological studies of $V$. parahaemolyticus infections and used for the analysis of genetic diversity. As a relatively simple method, ERIC-PCR was easier to perform than PFGE. It also was appropriate for the analysis of the large population of strains (Khan et al., 2002; Chen et al., 2012). Using this approach, the isolates were classified into five clusters when a 0.67 similarity cutoff value was used. However, we did not identify significant association between ERIC-PCR clusters and collection sites (source and provinces). Nevertheless, these result revealed high level of genetic diversity among V. parahaemolyticus isolates. Wong et al. (1999) showed a link between environment and likelihood of an outbreak (Mala et al., 2016). We also obtained similar results. MLST is also a useful method for typing strains because of its reproducibility and comparability. The approach has been commonly used for analysis of $V$. parahaemolyticus isolates (Guerrero et al., 2017). In this study, our isolates were grouped into five main clusters (I, II, III, IV, and V). MLST analysis revealed that the same source strains were in the same clusters (III, IV, and V) and suggested that these isolates have similar genotypes. As previously reported, the same ST types generally have the same serotype (Lüdeke et al., 2015). Multiple ST types were identified in our research. For example, ST291, ST300, and ST396 were identified in a public database as environmental isolates from China; ST1228, ST1251, and ST1231 were isolated from RTE foods; ST40 and ST162 were separated from environmental and clinical samples from the United States. There were also some clinical strains identified such as ST112 and ST739. ST212, which was isolated from clinical samples in Peru, showed greater evolutionary distance compared with other strains in our study. MLST analysis confirmed the genetic relatedness and genetic diversity occurring within those strains. Thus, ERIC-PCR and MLST are useful as phylogenetic research tools and as important methods for investigating outbreaks of $V$. parahaemolyticus.

Diarrhea due to the seafood borne pathogen $V$. parahaemolyticus has been a longstanding problem. In general, we provided the first comprehensive research on $V$. parahaemolyticus isolates from seafood in South China in both the summer and the winter by describing the prevalence, antibiotic resistance phenotypes, virulence, and molecular diversity. Our study showed that the prevalence of $V$. parahaemolyticus is $19.44 \%$. The percentages of the isolates possessing $t d h$ or trh were 8.16 and $12.24 \%$, respectively. The antimicrobial-resistance patterns showed that ampicillinresistance was widespread (79.59\%). ERIC-PCR and MLST typing showed genetic relatedness and genetic diversity within these isolates. As seafood is a common and popular food choice in China, our findings should serve as a reference for understanding antibiotic-resistant trends in V. parahaemolyticus 
strains, providing information for evaluating this bacteria at the level of consumption, and establishing the appropriate monitoring programs, which are important in ensuring the safety of seafood.

\section{AUTHOR CONTRIBUTIONS}

YY finished the experiments and wrote the article. HY gave the idea and experimental support. JX and HL provided assistance and guidance throughout the research. ST and YC helped to finish the experiments. All authors assisted in writing the article and manuscript editing.

\section{REFERENCES}

Banerjee, S. K., Kearney, A. K., Nadon, C. A., Peterson, C. L., Tyler, K., Bakouche, L., et al. (2014). Phenotypic and genotypic characterization of Canadian clinical isolates of Vibrio parahaemolyticus collected from 2000 to 2009. J. Clin. Microbiol. 52, 1081-1088. doi: 10.1128/JCM.03047-13

Chen, J., Zhang, R., Qi, X., Zhou, B., Wang, J., Chen, Y., et al. (2017). Epidemiology of foodborne disease outbreaks caused by Vibrio parahaemolyticus during 2010-2014 in Zhejiang Province, China. Food Control 77, 110-115. doi: 10.1016/j.foodcont.2017.02.004

Chen, W., Xie, Y., Xu, J., Wang, Q., Gu, M., Yang, J., et al. (2012). Molecular typing of Vibrio parahaemolyticus isolates from the middle-east coastline of China. Int. J. Food Microbiol. 153, 402-412. doi: 10.1016/j.ijfoodmicro.2011. 12.001

Cho, T., Kim, N., Kim, S., Song, J., and Rhee, M.-S. (2016). Survival of foodborne pathogens (Escherichia coli O157: H7, Salmonella Typhimurium, Staphylococcus aureus, Listeria monocytogenes, and Vibrio parahaemolyticus) in raw readyto-eat crab marinated in soy sauce. Int. J. Food Microbiol. 238, 50-55. doi: 10.1016/j.ijfoodmicro.2016.08.041

CLSI (2012). Methods for Antimicrobial Dilution and Disk Susceptibility Testing of Infrequently. Isolated or Fastidious Bacteria. Approved Standard M45-A. Wayne, PA: Clinical and Laboratory Standards Institute.

Daimei, C., and Dai, C. (2016). The present situation and countermeasures of antibiotic use in rural aquaculture industry - taking Zhangjiajie as an example [J]. Mod. Agric. Technol. 18, 246-250.

Daniels, N. A., Mackinnon, L., Bishop, R., Altekruse, S., Ray, B., Hammond, R. M., et al. (2000). Vibrio parahaemolyticus Infections in the United States, 1973-1998. J. Infect. Dis. 181, 1661-1666. doi: 10.1086/315459

Elexson, N., Afsah-Hejri, L., Rukayadi, Y., Soopna, P., Lee, H. Y., Zainazor, T. C. T., et al. (2014). Effect of detergents as antibacterial agents on biofilm of antibiotics-resistant Vibrio parahaemolyticus isolates. Food Control 35, 378-385. doi: 10.1016/j.foodcont.2013.07.020

Elmahdi, S., Dasilva, L. V., and Parveen, S. (2016). Antibiotic resistance of Vibrio parahaemolyticus and Vibrio vulnificus in various countries: a review. Food Microbiol. 57, 128-134. doi: 10.1016/j.fm.2016.02.008

Fujino, T., Okuno, Y., Nakada, D., Aoyama, A., Fukai, K., Mukai, T., et al. (1953). On the bacteriological examination of shirasu-food poisoning. Med. J. Osaka Univ. 4, 299-304.

Gonzálezescalona, N., Martinezurtaza, J., Romero, J., Espejo, R. T., Jaykus, L. A., and Depaola, A. (2008). Determination of molecular phylogenetics of Vibrio parahaemolyticus strains by multilocus sequence typing. J. Bacteriol. 190, 2831-2840. doi: 10.1128/JB.01808-07

Guerrero, A., Liceanavarro, A. F., Wongchang, I., and Gonzálezsánchez, R. (2017). Genetic analysis of Vibrio parahaemolyticus O3:K6 strains that have been isolated in Mexico since 1998. PLOS ONE 12:e0169722. doi: 10.1371/journal. pone. 0169722

Gutierrez West, C. K., Klein, S. L., and Lovell, C. R. (2013). High frequency of virulence factor genes tdh, trh, and th in Vibrio parahaemolyticus strains isolated from a pristine estuary. Appl. Environ. Microbiol. 79, 2247-2252. doi: 10.1128/AEM.03792-12

\section{ACKNOWLEDGMENTS}

Our work was supported by research grants from the National Natural Science Foundation of Guangdong, China (2017A030310642) and Science and Technology Planning Project of Guangdong Province, China (2016A020210141).

\section{SUPPLEMENTARY MATERIAL}

The Supplementary Material for this article can be found online at: https://www.frontiersin.org/articles/10.3389/fmicb. 2017.02566/full\#supplementary-material

Honda, T., and Iida, T. (1993). The pathogenicity of Vibrio parahaemolyticus and the role of the thermostable direct haemolysin and related haemolysins. Rev. Med. Microbiol. 4, 106-113. doi: 10.1097/00013542-199304000-00006

Jolley, K. A., Chan, M.-S., and Maiden, M. C. (2004). mlstdbNet-distributed multi-locus sequence typing (MLST) databases. BMC Bioinformatics 5:86. doi: 10.1186/1471-2105-5-86

Jones, J. L., Lüdeke, C. H., Bowers, J. C., Garrett, N., Fischer, M., Parsons, M. B., et al. (2012). Biochemical, serological, and virulence characterization of clinical and oyster Vibrio parahaemolyticus isolates. J. Clin. Microbiol. 50, 2343-2352. doi: 10.1128/JCM.00196-12

Joseph, S. W., Colwell, R. R., and Kaper, J. B. (1982). Vibrio parahaemolyticus and related halophilic Vibrios. Crit. Rev. Microbiol 10, 77-124. doi: 10.3109/ 10408418209113506

Jun, J. W., Kim, J. H., Choresca, C. H. Jr., Shin, S. P., Han, J. E., Han, S. Y., et al. (2012). Isolation, molecular characterization, and antibiotic susceptibility of Vibrio parahaemolyticus in Korean seafood. Foodborne Pathog. Dis. 9, 224-231. doi: 10.1089/fpd.2011.1018

Kang, C. H., Shin, Y. J., Kim, W. R., Kim, Y. G., Song, K. C., Oh, E. G., et al. (2016). Prevalence and antimicrobial susceptibility of Vibrio parahaemolyticus isolated from oysters in Korea. Environ. Sci. Pollut. Res. 23, 1-9. doi: 10.1007/s11356015-5650-9

Khan, A. A., McCarthy, S., Wang, R. F., and Cerniglia, C. E. (2002). Characterization of United States outbreak isolates of Vibrio parahaemolyticus using enterobacterial repetitive intergenic consensus (ERIC) PCR and development of a rapid PCR method for detection of O3:K6 isolates. FEMS Microbiol. Lett. 206, 209-214. doi: 10.1111/j.1574-6968.2002.tb1 1011.X

Kim, Y. B., Okuda, J., Matsumoto, C., Takahashi, N., Hashimoto, S., and Nishibuchi, M. (1999). Identification of Vibrio parahaemolyticus strains at the species level by PCR targeted to the toxR gene. J. Clin. Microbiol. 37, 1173-1177.

Leoni, F., Talevi, G., Masini, L., Ottaviani, D., and Rocchegiani, E. (2016). Trh $(t d h-/ t r h+)$ gene analysis of clinical, environmental and food isolates of Vibrio parahaemolyticus as a tool for investigating pathogenicity. Int. J. Food Microbiol. 225, 43-53. doi: 10.1016/j.ijfoodmicro.2016.02.016

Letchumanan, V., Pusparajah, P., Tan, T. H., Yin, W. F., Lee, L. H., and Chan, K. G. (2015a). Occurrence and antibiotic resistance of Vibrio parahaemolyticus from shellfish in Selangor, Malaysia. Front. Microbiol. 6:1417. doi: 10.3389/fmicb. 2015.01417

Letchumanan, V., Yin, W. F., Lee, L. H., and Chan, K. G. (2015b). Prevalence and antimicrobial susceptibility of Vibrio parahaemolyticus isolated from retail shrimps in Malaysia. Front. Microbiol. 6:33. doi: 10.3389/fmicb.2015. 00033

Lin, Z., Kumagai, K., Baba, K., Mekalanos, J., and Nishibuchi, M. (1993). Vibrio parahaemolyticus has a homolog of the Vibrio cholerae toxRS operon that mediates environmentally induced regulation of the thermostable direct hemolysin gene. J. Bacteriol. 175, 3844-3855. doi: 10.1128/jb.175.12.3844-3855. 1993

Lopatek, M., Wieczorek, K., and Osek, J. (2015). Prevalence and antimicrobial resistance of Vibrio parahaemolyticus isolated from raw shellfish in Poland. J. Food Prot. 78, 1029-1033. doi: 10.4315/0362-028X.JFP-14-437 
Lüdeke, C. H., Gonzalez-Escalona, N., Fischer, M., and Jones, J. L. (2015). Examination of clinical and environmental Vibrio parahaemolyticus isolates by multi-locus sequence typing (MLST) and multiple-locus variable-number tandem-repeat analysis (MLVA). Front. Microbiol. 6:564. doi: 10.3389/fmicb. 2015.00564

Mahoney, J. C., Gerding, M. J., Jones, S. H., and Whistler, C. A. (2010). Comparison of the pathogenic potentials of environmental and clinical Vibrio parahaemolyticus strains indicates a role for temperature regulation in virulence. Appl. Environ. Microbiol. 76, 7459-7465. doi: 10.1128/AEM. 01450-10

Mala, W., Alam, M., Angkititrakul, S., Wongwajana, S., Lulitanond, V., Huttayananont, S., et al. (2016). Serogroup, virulence, and molecular traits of Vibrio parahaemolyticus isolated from clinical and cockle sources in northeastern Thailand. Infect. Genet. Evol. 39, 212-218. doi: 10.1016/j.meegid. 2016.01.006

Marshall, S., Clark, C. G., Wang, G., Mulvey, M., Kelly, M. T., and Johnson, W. M. (1999). Comparison of molecular methods for typing Vibrio parahaemolyticus. J. Clin. Microbiol. 37, 2473-2478.

Matsuda, S., Kodama, T., Okada, N., Okayama, K., Honda, T., and Iida, T. (2010). Association of Vibrio parahaemolyticus thermostable direct hemolysin with lipid rafts is essential for cytotoxicity but not hemolytic activity. Infect. Immun. 78, 603-610. doi: 10.1128/IAI.00946-09

Miller, R., Carson, J., Dalsgaard, I., Gaunt, P., Gieseker, C., Hawke, J., et al. (2014). CLSI Performance Standards for Antimicrobial Susceptibility Testing of Bacteria Isolated from Aquatic Animals; Second Information Supplement. Wayne, PA: CLSI.

Odeyemi, O. A., and Stratev, D. (2016). Occurrence of antimicrobial resistant or pathogenic Vibrio parahaemolyticus in seafood. A mini review. Rev. Méd. Vét. 167, 93-98.

Ottaviani, D., Leoni, F., Talevi, G., Masini, L., Santarelli, S., Rocchegiani, E., et al. (2013). Extensive investigation of antimicrobial resistance in Vibrio parahaemolyticus from shellfish and clinical sources, Italy. Int. J. Antimicrob. Agents 42, 191-193. doi: 10.1016/j.ijantimicag.2013. 05.003

Raghunath, P. (2015). Roles of thermostable direct hemolysin (TDH) and TDHrelated hemolysin (TRH) in Vibrio parahaemolyticus. Front. Microbiol. 5:805. doi: $10.3389 /$ fmicb. 2014.00805

Rohlf, F. J. (2000). Ntsys-Pc: Numerical Taxonomy and Multivariate Analysis System, Version 2.2. Setauket, NY: Exeter Publishing.

Shaw, K. S., Rosenberg Goldstein, R. E., He, X., Jacobs, J. M., Crump, B. C., and Sapkota, A. R. (2014). Antimicrobial susceptibility of Vibrio vulnificus and Vibrio parahaemolyticus recovered from recreational and commercial areas of Chesapeake Bay and Maryland Coastal Bays. PLOS ONE 9:e89616. doi: 10.1371/ journal.pone.0089616

Shen, X., Liu, W., and Liu, C. (2010). "Effects of cold storage and thermal treatment on growth and survival of pathogenic Vibrio parahaemolyticus," in Proceedings of the International Conference on Bioinformatics and Biomedical Technology, Chengdu, 371-373.

Shirai, H., Ito, H., Hirayama, T., Nakamoto, Y., Nakabayashi, N., Kumagai, K., et al. (1990). Molecular epidemiologic evidence for association of thermostable direct hemolysin (TDH) and TDH-related hemolysin of Vibrio parahaemolyticus with gastroenteritis. Infect. Immun. 58, 3568-3573.

Su, Y. C., and Liu, C. (2007). Vibrio parahaemolyticus: a concern of seafood safety. Food Microbiol. 24, 549-558. doi: 10.1016/j.fm.2007.01.005

Takahashi, A., Kenjyo, N., Imura, K., Myonsun, Y., and Honda, T. (2000). $\mathrm{Cl}$ - secretion in colonic epithelial cells induced by the Vibrio parahaemolyticus hemolytic toxin related to thermostable direct hemolysin. Infect. Immun. 68, 5435-5438. doi: 10.1128/IAI.68.9.5435-5438.2000

Tamura, K., Stecher, G., Peterson, D., Filipski, A., and Kumar, S. (2013). MEGA6: molecular evolutionary genetics analysis version 6.0. Mol. Biol. Evol. 30, 2725-2729. doi: 10.1093/molbev/mst197

Tan, C. W., Malcolm, T. T. H., Kuan, C. H., Thung, T. Y., Chang, W. S., Loo, Y. Y., et al. (2017). Prevalence and antimicrobial susceptibility of Vibrio parahaemolyticus isolated from short mackerels (Rastrelliger brachysoma) in Malaysia. Front. Microbiol. 8:1087. doi: 10.3389/fmicb.2017. 01087

Tan, L. T., Chan, K. G., Lee, L. H., and Goh, B. H. (2016). Streptomyces bacteria as potential probiotics in aquaculture. Front. Microbiol. 7:79. doi: 10.3389/fmicb. 2016.00079

West, C. K. G., Klein, S. L., and Lovell, C. R. (2013). High frequency of virulence factor genes tdh, trh, and th in Vibrio parahaemolyticus strains isolated from a pristine estuary. Appl. Environ. Microbiol. 79, 2247-2252. doi: 10.1128/AEM. 03792-12

Wilson, B. A., and Salyers, A. A. (2003). Is the evolution of bacterial pathogens an out-of-body experience? Trends Microbiol. 11, 347-350.

Wong, H. C., Chen, M. C., Liu, S. H., and Liu, D. P. (1999). Incidence of highly genetically diversified Vibrio parahaemolyticus in seafood imported from Asian countries. Int. J. Food Microbiol. 52, 181-188. doi: 10.1016/S0168-1605(99) 00143-9

Wong, H. C., and Lin, C. H. (2001). Evaluation of typing of Vibrio parahaemolyticus by three PCR methods using specific primers. J. Clin. Microbiol. 39, 4233-4240.

World Health Organization (2014). EN JEMRA Joint FAO/WHO Expert Meetings on Microbiological Risk Assessment. Geneva: WHO.

Xie, T., Wu, Q., Xu, X., Zhang, J., and Guo, W. (2015). Prevalence and population analysis of Vibrio parahaemolyticus in aquatic products from South China markets. FEMS Microbiol. Lett. 362:fnv178. doi: 10.1093/femsle/fnv178

Xie, T., Wu, Q., Zhang, J., Xu, X., and Cheng, J. (2017). Comparison of Vibrio parahaemolyticus isolates from aquatic products and clinical by antibiotic susceptibility, virulence, and molecular characterisation. Food Control 71, 315-321. doi: 10.1016/j.foodcont.2016.06.046

Xie, T., Xu, X., Wu, Q., Zhang, J., and Cheng, J. (2016). Prevalence, molecular characterization, and antibiotic susceptibility of Vibrio parahaemolyticus from ready-to-eat foods in China. Front. Microbiol. 7:549. doi: 10.3389/fmicb.2016. 00549

Xu, X., Cheng, J., Wu, Q., Zhang, J., and Xie, T. (2016). Prevalence, characterization, and antibiotic susceptibility of Vibrio parahaemolyticus isolated from retail aquatic products in North China. BMC Microbiol. 16:32. doi: 10.1186/s12866-016-0650-6

Yang, Z. Q., Jiao, X. A., Zhou, X. H., Cao, G. X., Fang, W. M., and Gu, R. X. (2008). Isolation and molecular characterization of Vibrio parahaemolyticus from fresh, low-temperature preserved, dried, and salted seafood products in two coastal areas of eastern China. Int. J. Food Microbiol. 125, 279-285. doi: 10.1016/j.ijfoodmicro.2008.04.007

Yano, Y., Hamano, K., Satomi, M., Tsutsui, I., Ban, M., and Aue-Umneoy, D. (2014). Prevalence and antimicrobial susceptibility of Vibrio species related to food safety isolated from shrimp cultured at inland ponds in Thailand. Food Control 38, 30-36. doi: 10.1016/j.foodcont.2013.09.019

Yu, Q., Niu, M., Yu, M., Liu, Y., Wang, D., and Shi, X. (2015). Prevalence and antimicrobial susceptibility of Vibrio parahaemolyticus isolated from retail shellfish in Shanghai. Food Control 60, 263-268. doi: 10.1016/j.foodcont.2015. 08.005

Zhao, F., Zhou, D. Q., Cao, H. H., Ma, L. P., and Jiang, Y. H. (2011). Distribution, serological and molecular characterization of Vibrio parahaemolyticus from shellfish in the eastern coast of China. Food Control 22, 1095-1100. doi: 10.1016/j.foodcont.2010.12.017

Conflict of Interest Statement: The authors declare that the research was conducted in the absence of any commercial or financial relationships that could be construed as a potential conflict of interest.

Copyright (C) 2017 Yang, Xie, Li, Tan, Chen and Yu. This is an open-access article distributed under the terms of the Creative Commons Attribution License (CC BY). The use, distribution or reproduction in other forums is permitted, provided the original author(s) or licensor are credited and that the original publication in this journal is cited, in accordance with accepted academic practice. No use, distribution or reproduction is permitted which does not comply with these terms. 